\title{
3D T1-weighted self-gated cardiac MRI for assessing myocardial infarction in mouse models
}

\author{
Xiaoyong Zhang ${ }^{1,2}$, Hanwei Chen ${ }^{3}$, Zijun Wei ${ }^{2}$ Fei Yan², Shi Su², Yanchun Zhu², Bensheng Qiu' ${ }^{1}$ Xin Liu²*, \\ Zhaoyang Fan ${ }^{4}$, Guoxi Xie ${ }^{2}$
}

From 19th Annual SCMR Scientific Sessions

Los Angeles, CA, USA. 27-30 January 2016

\section{Background}

Mouse models with mycardial infarction (MI) have been intensively used to investigate the cardiac remodeling and functional change [1,2]. MRI with ECG triggering and respiratory gating (or breath-holding) is commonly used to assess MI due to its noninvasive nature. However, the manipulations of external ECG triggering and respiratory gating are cumbersome due to the small size of mouse. To address this issue, a 3D self-gating (SG) MR technique with stack-of-stars sampling trajectories was proposed for retrospectively cardiac and respiratory-gated MI imaging in mouse models [3].

Methods: Technical Design: MR data was acquired by a T1-weighted GRE sequence with stack-of-stars sampling trajectories and a partition-first golden-angle reordering (Figure 1a). The centers of k-space lines acquired at the same angle from individual partitions were aggregated and used as an SG time point. The periodic respiratory and cardiac motions were then detected through an iterative filtering process on the SG time series according to the cardiac rate of 300 500 per minute and respiration rate of 70 110 per minute. After resorting the imaging data into appropriate cardiac and respiratory phases, motion-artifact-free were finally reconstructed.

Experiments: The SG technique was preliminarily validated on 5 mice with MI induction and all MR scans were performed on a $3 \mathrm{~T}$ scanner (Siemens Tim Trio, Germany) with a customized 4-channal mouse coil. Typical imaging parameters for the ungated GRE sequence included: flip angle $=18^{\circ}, \mathrm{TR}=4.2 \mathrm{~ms}, \mathrm{TE}=$ $2.4 \mathrm{~ms}$, spatial resolution $=0.6 \times 0.6 \times 1.5 \mathrm{~mm}^{3}$, bandwidth $=620 \mathrm{~Hz} /$ Pixel, partition number $=12$, and $\mathrm{a}$

${ }^{2}$ Shenzhen Institutes of Advanced Technology, Shenzhen, China
Full list of author information is available at the end of the article total number of 3200 projections were continuously collected, corresponding to a fixed scan time of $3 \mathrm{~min}$. An amount of $0.5 \mathrm{ml}$ gadolinium contrast agent with concentration of $0.5 \mathrm{mmol} / \mathrm{ml}$ (Consun Pharmaceutical Group Limited, GuangZhou, China) was injected to enhance the MI. The mice were sacrificed immediately after MRI for histological analysis and comparison to the MR results.

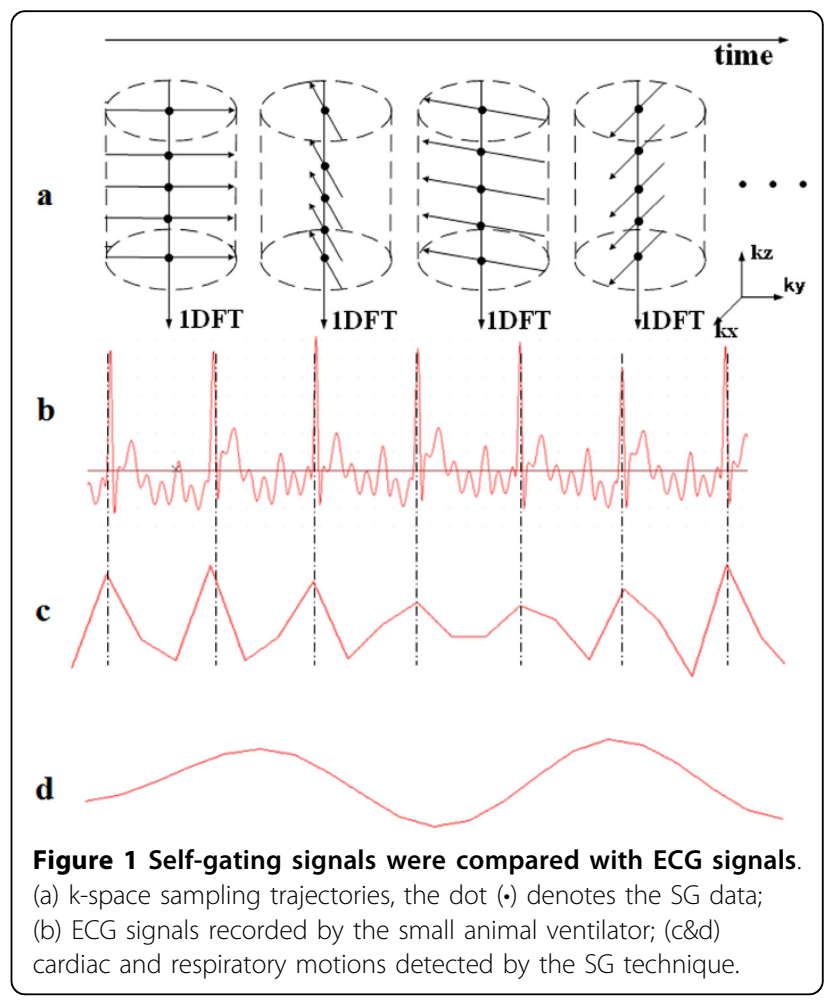




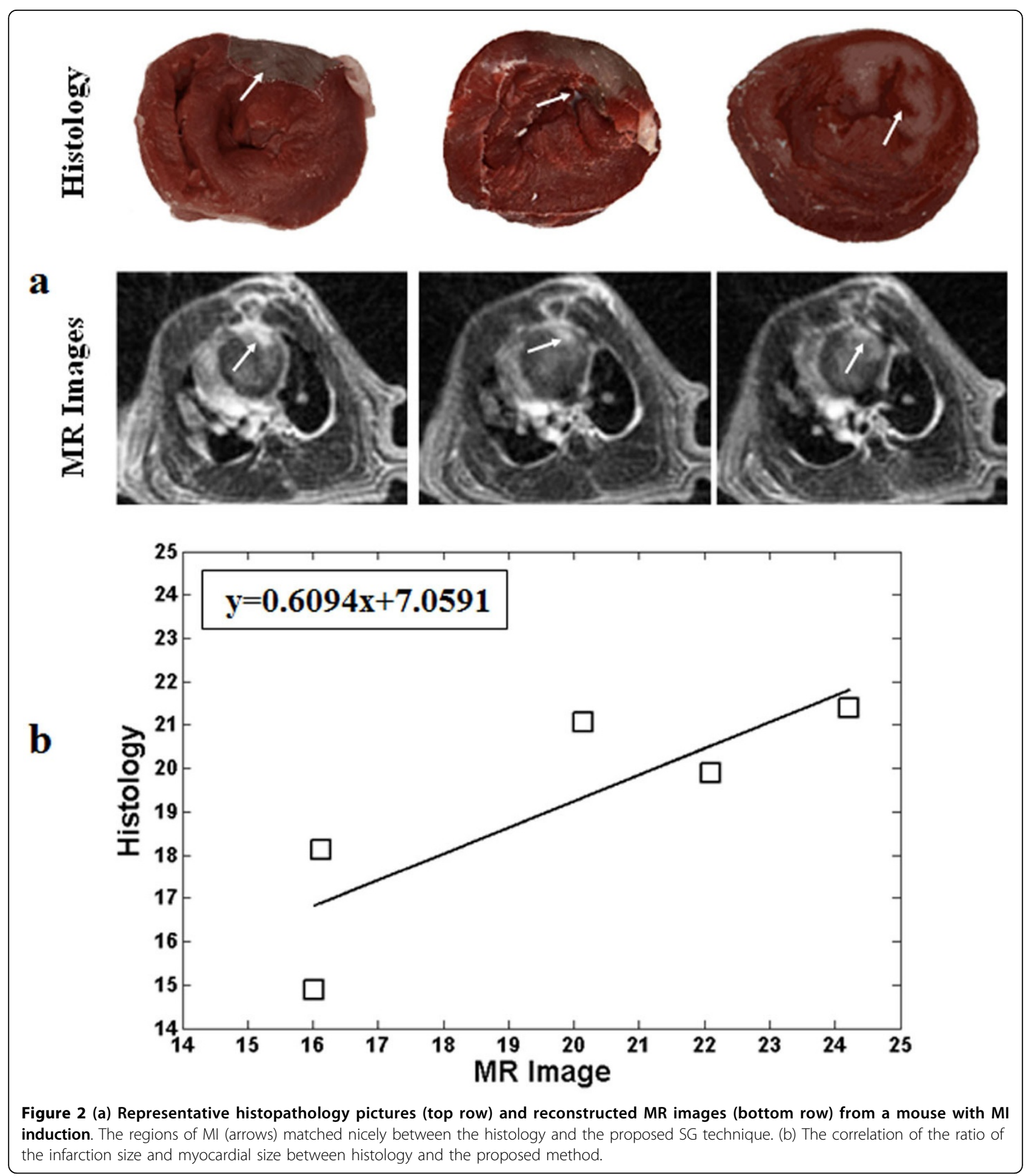

\section{Results}

All MR scans were successfully conducted. The cardiac motion detected by the SG technique matched nicely to the ECG signals recorded by a small animal ventilator (Chengdu Taimeng Software Co.LTD, Chengdu, China) (Fig. 1b\&1c). And the MI regions detected by the SG technique were also matched well to the histopathology analysis results (Fig. 2).

\section{Conclusions}

A 3D SG technique was developed for assessing MI in mouse model. The preliminary in vivo study has 
demonstrated that the technique can correctly detect the MI, which may outperform the conventional MR techniques with ECG-triggering and respiratory gating.

\section{Authors' details}

'University of Science and Technology of China, Hefei, China. ${ }^{2}$ Shenzhen Institutes of Advanced Technology, Shenzhen, China. ${ }^{3}$ Guangzhou Panyu Central Hospital, Guangzhou, China. ${ }^{4}$ Cedars-Sinai Medical Center, Los Angeles, CA, USA.

Published: 27 January 2016

\section{References}

1. Lutgens $\mathrm{E}$, et al: Cardiovascular research 1999, 41(3):586-593.

2. Hofmann U, et al: Circulation 2012, 125(13):1652-1663.

3. Liu J, et al: MRM 2010, 63(5):1230-1237.

doi:10.1186/1532-429X-18-S1-Q49

Cite this article as: Zhang et al:: 3D T1-weighted self-gated cardiac MRI

for assessing myocardial infarction in mouse models. Journal of

Cardiovascular Magnetic Resonance 2016 18(Suppl 1):Q49.

Submit your next manuscript to BioMed Central and take full advantage of:

- Convenient online submission

- Thorough peer review

- No space constraints or color figure charges

- Immediate publication on acceptance

- Inclusion in PubMed, CAS, Scopus and Google Scholar

- Research which is freely available for redistribution

Submit your manuscript at www.biomedcentral.com/submit 